\title{
Choosing the Most Distally Located Digital Artery Perforator as the Pedicle of the Homodigital Island Flap
}

\author{
Ersen $B^{*}$ \\ Plastic Aesthetic and Reconstructive Surgery Department, Dr. Munif Islamoglu Kastamonu State \\ Hospital, Kastamonu, Turkey
}

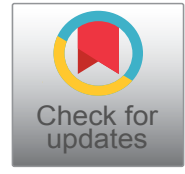

*Corresponding author: Ersen B, Plastic Aesthetic and Reconstructive Surgery Department, Dr. Munif Islamoglu Kastamonu State Hospital, Odunluk Mahallesi, Mihraplı Cad. Orhaneli Yolu Beşevler Kavşağı No: 6, 16110 Nilüfer/Bursa, Turkey, Tel: 00-90-533-422-4801

It is difficult to cover distal defects of the dorsum, ulnar or radial sides of the finger like those of the nail matrix or a terminal extensor tendon, because there is less soft tissue in this region. Appropriate reconstruction should preserve the aesthetic appearance, provide sensate coverage without pain in important anatomical regions, and minimize donor-site morbidity [1].

Digital homodigital island flap (DHI) flaps are perforator flaps based on the dorsal branch the digital artery. Koshima, et al. [2] originally reported the utility of these flaps for fingertip reconstruction. The distribution of the dorsal branches of the digital artery is relatively constant. At the level of the middle and distal thirds of the proximal phalanx, the middle third of the middle phalanx, and the distal interphalangeal joint, the digital artery branches off the four largest dorsal branches of the digital artery [3]. Based on these dorsal branches of the digital artery, the different dorsal digital flaps can be selected for various reconstructive requirements [4].

In this case, the most distally located digital artery perforator was chosen as the pedicle of the flap. The advantages of this flap are that it is mobile, has a small skin island and is less invasive compared with other flaps reported for use in the reconstruction of the finger. It was considered that thumb reconstruction with a $\mathrm{DHI}$ flap by choosing the most distally located digital artery perforator is a rare entity.

A 42-year-old male patient sustained a sharp injury to the right thumb and the dorsum of the hand. He presented with a full thickness soft tissue damage to the ulnar side of the distal thumb. It was seen that there was a $1.5 \mathrm{~cm} \times 1.5 \mathrm{~cm}$ tissue defect on the ulnar side of the distal thumb. Bone component was visible. The circulation of the finger was intact and no bone fracture was seen in the X-ray.

The operation was performed under local anesthe-

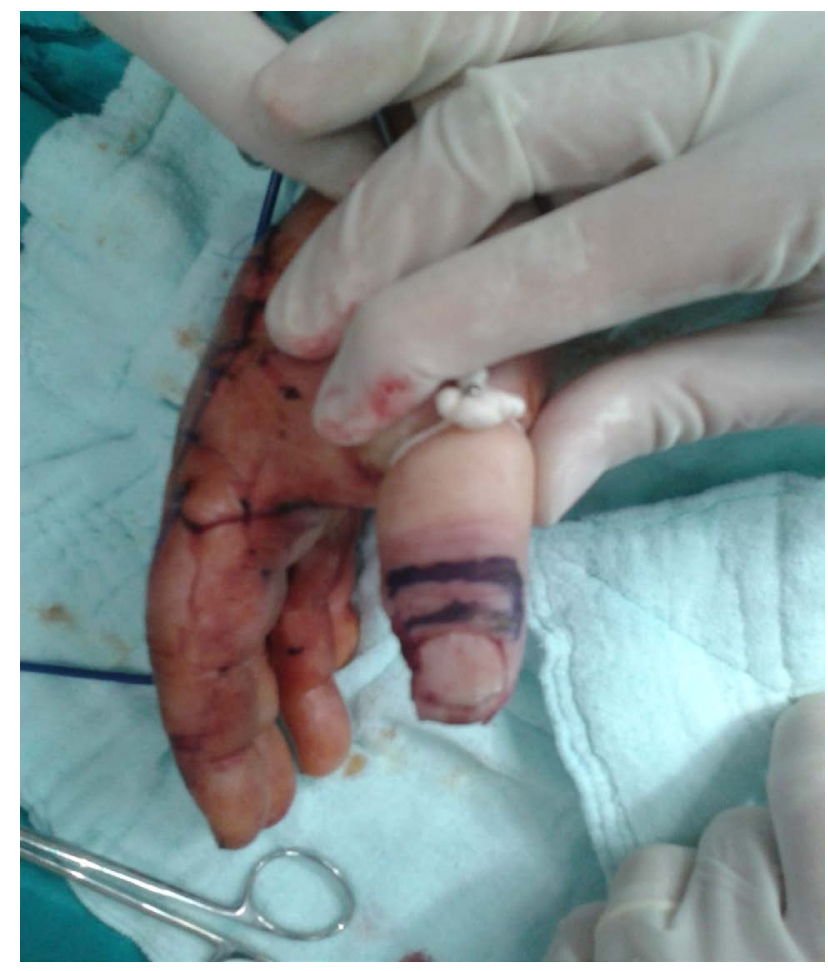

Figure 1: DHI flap was planned from the proximal dorsal area of the distal phalanges of the thumb. 
sia with proper preparation in the operation theatre. $A$ $1.5 \mathrm{~cm} \times 1.5 \mathrm{~cm}$ DHI flap was planned from the proximal dorsal area of the distal phalanges of the thumb (Figure 1). The designed DHI flap was based on the most distally located digital artery perforator. The flap was elevated including a thin layer of fascia and transposed to cover the tissue defect on the ulnar side of the distal thumb. The paratenon of the extensor tendon was left intact during the flap elevation. Flap donor site healed by secondary intention. Postoperatively, the color of the flap was excellent. The flap survived completely (Figure 2). On the $3^{\text {th }}$ month post-operatively excellent donor site morbidity was seen (Figure 3).

To repair small to moderate defects, a variety of flaps have been described, allowing the use of a specific flap for each reconstructive situation $[5,6]$. The $\mathrm{DHI}$ flap is an axial fasciocutaneous island flap based on the dorsal branches of the digital artery. Venous drainage is through tiny venules and capillaries embedded in the perivascular fat of the pedicle [4]. As described in previous anatomical studies, the distribution of the dorsal branches of the digital artery is relatively constant [7].

According to Braga, Silvia, et al., the dorsal perforator arising directly from the proper digital artery seems to be equal to the fifth cutaneous dorsal branch with a diameter of 0.2-0.4 $\mathrm{mm}$ and a location 9-13 $\mathrm{mm}$ distal to the PIP joint. These perforating branches of the proper digital artery can be found running from the volar to the dorsal side to nourish the skin [7]. This one-stage local flap is preferred to multistage regional flaps because of

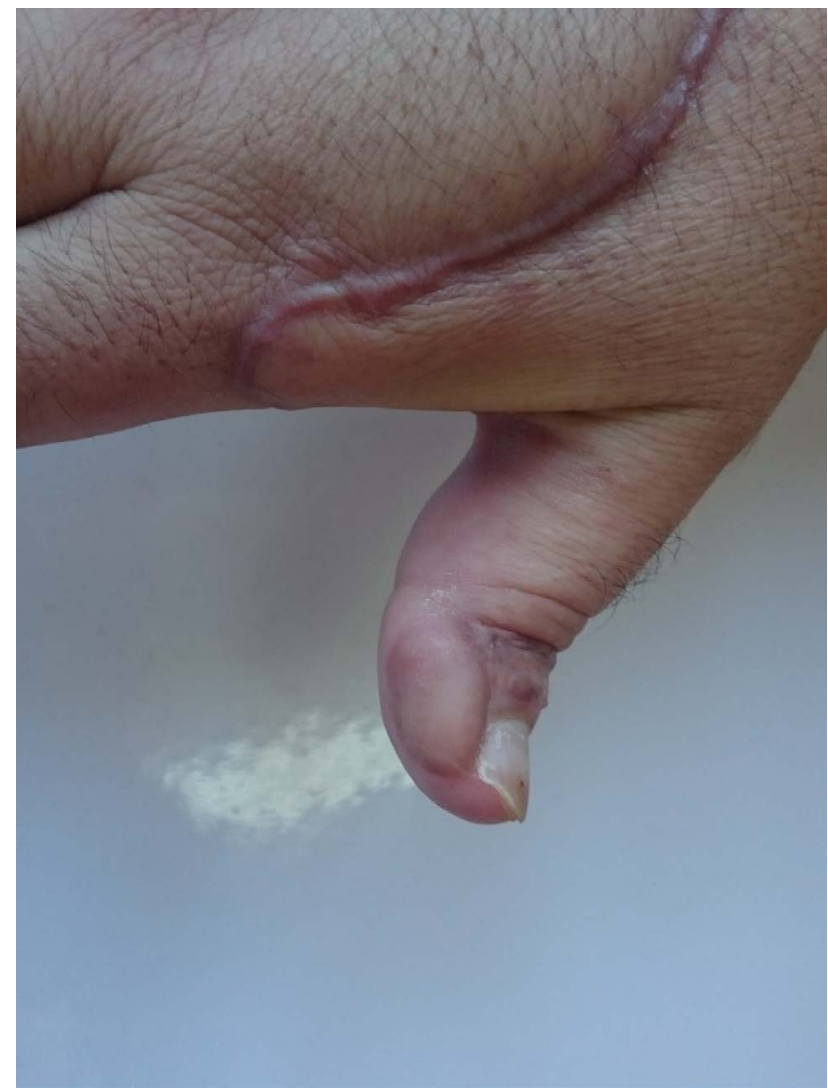

Figure 2: The flap survived completely. shorter hospital stays, lower cost, and minimal disability time [8].

Using the dorsal homodigital island flap, the entire dorsum of the middle and proximal phalanges can be used as the donor based on the different levels of the dorsal branches of the digital artery. This important feature makes it more versatile for reconstructing defects in different regions of the finger, especially for simultaneously reconstructing small to moderate defects in multiple fingers [9].

The literature dictates that the homodigital donor-site morbidity is a major concern when raising a dorsal homodigital island flap. Skin graft contracture and extensor tendon adhesion are inevitable, but early rehabilitation with the help of a physical therapist can be less prone to these complications [10]. The donor site was healed by secondary intention in this case without any healing or range of motion problems. It was considered that the location of the donor site was the main reason for problem free healing. Tendon gliding is minimal at the distal portion of the finger therefore donor site suffered from less disruption.

The advantages of the dorsal homodigital island flap include a single-stage procedure, simultaneous coverage of multiple-finger defects, sensory reconstruction in important anatomical regions, and minimal donor-site morbidity. Limited flap size is its major disadvantage.

It was considered that choosing the most distally located perforator of the digital artery as a pedicle for $\mathrm{DHI}$ flap is a rare entity.

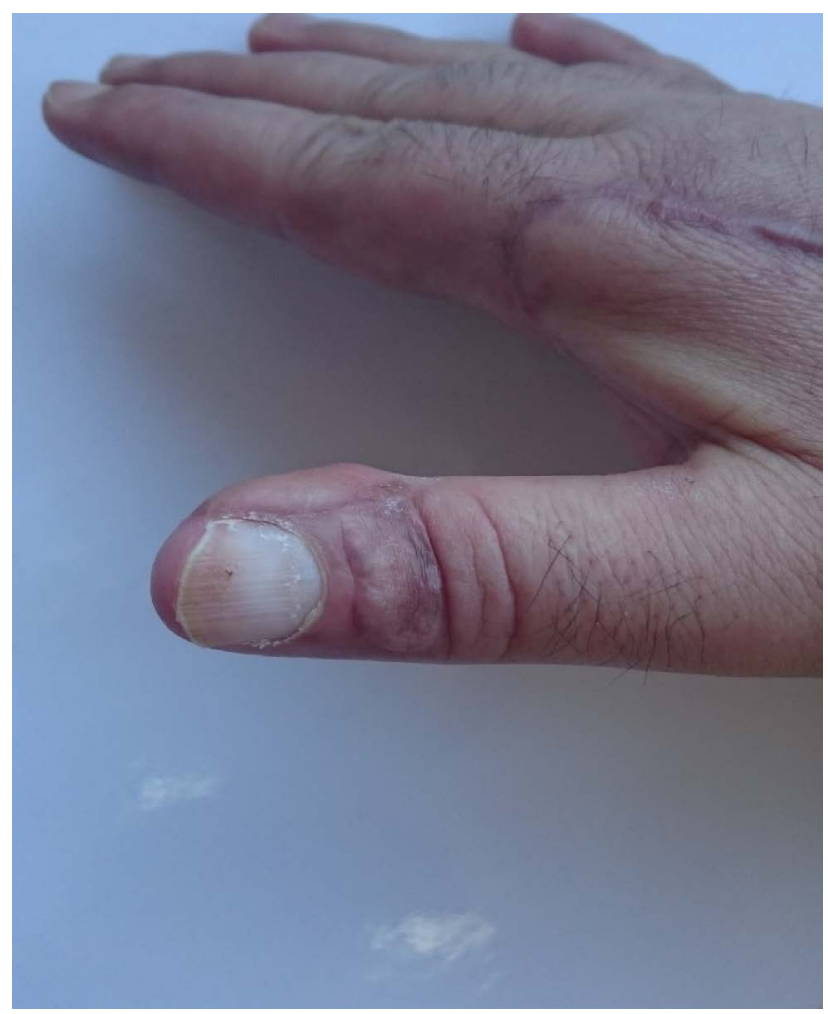

Figure 3: Post-operatively excellent donor site morbidity was seen. 


\section{Conflict of Interest}

None.

\section{Patient Consent}

The written consent for the use of his images was signed by the patient.

\section{Ethical Standards}

This study has been approved by the appropriate ethics committee and has therefore been performed in accordance with the ethical standards set forth in the 1964 Declaration of Helsinki and its later amendments.

\section{References}

1. Franz L, Michael B, Alfred B, Pallua N (2002) Sensory reconstruction of the fingertip using the bilaterally innervated sensory cross-finger flap. Plast Reconstr Surg 109: 988-993.

2. Koshima I, Urushibara K, Fukuda N, Ohkochi M, Nagase T, et al. (2006) Digital artery perforator flaps for fingertip reconstructions. Plast Reconstr Surg 118: 1579-1584.

3. Li YF, Cui SS (2005) Innervated reverse island flap based on the end dorsal branch of the digital artery: Surgical technique. J Hand Surg Am 30: 1305-1309.
4. Kawakatsu M, Ishikawa K (2010) Dorsal digital perforator flap for reconstruction of distal dorsal finger defects. J Plast Reconstr Aesthet Surg 63: e46-e50.

5. Sano K, Aoki S, Koike S, Hyakusoku H (2007) Delayed extended "midthenar" flap for reconstruction of total fingertip avulsion injury and a proposal of ideal postoperative immobilization for a palmar flap. Ann Plast Surg 58: 116119.

6. Teoh LC, Tay SC, Yong FC, Tan SH, Khoo DB (2003) Heterodigital arterialized flaps for large finger wounds: Results and indications. Plast Reconstr Surg 111: 19051913.

7. Strauch B, de Moura W (1990) Arterial system of the fingers. J Hand Surg Am 15: 148-154.

8. Pelissier P, Casoli V, Bakhach J, Martin D, Baudet J (1999) Reverse dorsal digital and metacarpal flaps: A review of 27 cases. Plast Reconstr Surg 103: 159-165.

9. Chen C, Tang P, Zhang X (2014) The dorsal homodigital island flap based on the dorsal branch of the digital artery: $A$ review of 166 cases. Plast Reconstr Surg 133: 519e-529e.

10. Shao X, Chen C, Zhang X, Yu Y, Ren D, et al. (2009) Coverage of fingertip defect using a dorsal island pedicle flap including both dorsal digital nerves. J Hand Surg Am 34: 1474-1481. 